$10,11,05$

\title{
Исследование влияния слабых магнитных полей на термодинамические свойства модели Поттса с числом состояний спина $q=4$ на гексагональной решетке
}

\author{
(C) М.К. Рамазанов ${ }^{1,2}$, А.К. Муртазаев ${ }^{1,2}$, М.А. Магомедов ${ }^{1,2}$, М.К. Мазагаева ${ }^{1}$, \\ M.P. Джамалудинов ${ }^{1}$ \\ ${ }^{1}$ Институт фризики Дагестанского федерального исследовательского центра РАН, \\ Махачкала, Россия \\ 2 Дагестанский фредеральный исследовательский центр РАН, \\ Махачкала, Россия \\ E-mail: sheikh77@mail.ru
}

Поступила в Редакцию 22 октября 2021 г.

В окончательной редакции 22 октября 2021 г.

Принята к публикации 24 октября 2021 г.

Репличным обменным алгоритмом метода Монте-Карло проведено исследование фазовых переходов и термодинамических свойств двумерной модели Поттса с числом состояний спина $q=4$ на гексагональной решетке в слабых магнитных полях. Исследования проведены для интервала величины магнитного поля $0.0 \leq H \leq 3.0$ с шагом 1.0. Установлено, что в рассмотренном интервале значений поля наблюдается фазовый переход первого рода.

Ключевые слова: фрустрации, фазовые переходы, метод Монте-Карло, модель Поттса.

DOI: 10.21883 /FTT.2022.02.51935.226

\section{1. Введение}

В последние годы в физике конденсированного состояния наблюдается повышенный интерес к исследованию влияния различных возмущающих факторов на фазовые переходы (ФП), магнитные структуры основного состояния, критические, магнитные и термодинамические свойства спиновых систем. На сегодняшний день вопрос о влиянии внешнего магнитного поля, взаимодействий вторых ближайших соседей, немагнитных примесей, тепловых и квантовых флуктуаций имеет принципиальное значение. Включение этих возмущающих факторов может привести к большому разнообразию фаз и ФП в магнитных спиновых системах [1-7]. Особый интерес имеет исследование влияния внешних факторов на спиновые системы с фрустрациями. Это связано с тем, что фрустрированные спиновые системы обладают свойствами отличными от соответствующих нефрустрированных систем. Фрустрированные системы обладают высокой чувствительностью к внешним возмущающим факторам. Внесение внешних возмущений в такие системы может привести к совершенно новому физическому поведению. В связи с этим, в данном исследовании нами изучается влияние слабых магнитных полей на характер ФП и термодинамические свойства спиновых систем с фрустрациями. При решении такого рода задач успешно используют различные решеточные модели, такие как модель Изинга, Поттса, Гейзенберга и др.

К настоящему моменту времени влияние внешних возмущающих факторов, в том числе и магнитного поля в модели Изинга и Гейзенберга достаточно хорошо изучено [8-13]. Совсем иначе обстоит дело с моделью Поттса. Модель Поттса является малоизученной. Интерес к этой модели обусловлен тем, что модель Поттса служит основой теоретического описания широкого круга физических свойств и явлений в физике конденсированных сред. К их числу относятся сложные анизотропные ферромагнетики кубической структуры, спиновые стекла, многокомпонентные сплавы и жидкие смеси. На основе модели Поттса с различным числом состояний спина могут быть описаны структурные ФП во многих материалах [13]. Работ, посвященных изучению влияния внешнего магнитного поля, как возмущающего фактора, на ФП и термодинамические свойства модели Поттса практически нет, и этот вопрос все еще остается открытым и малоизученным.

В связи с этим, в настоящей работе нами на основе метода Монте-Карло (МК) изучается влияние слабых магнитных полей на ФП и термодинамические свойства двумерной модели Поттса с числом состояний спина $q=4$ на гексагональной решетке с учетом обменных взаимодействий первых и вторых ближайших соседей. Данная модель интересна еще и тем, что значение $q=4$ является граничным значением интервала $2 \leq q \leq 4$, где наблюдается ФП второго рода и области значений $q>4$, в котором наблюдается ФП первого рода [14]. Исследования проводятся на основе современных методов и идей, что позволит получить ответ на ряд вопросов, связанных с характером и природой ФП фрустрированных спиновых систем. 


\section{2. Модель и метод исследования}

Гамильтониан модели Поттса с учетом взаимодействия первых и вторых ближайших соседей, а также внешнего магнитного поля имеет следующий вид:

$$
\begin{aligned}
H & =-J_{1} \sum_{\langle i, j,\rangle, i \neq j} S_{i} S_{j}-J_{2} \sum_{\langle i, j,\rangle, i \neq k} S_{i} S_{k}-H \sum_{\langle i\rangle} S_{i} \\
& =-J_{1} \sum_{\langle i, j,\rangle, i \neq j} \cos \theta_{i, j}-J_{2} \sum_{\langle i, j,\rangle, i \neq k} \cos _{i, k}-H \sum_{\langle i\rangle} S_{i}
\end{aligned}
$$

где $J_{1}$ и $J_{2}$ - параметры обменных ферро- $\left(J_{1}>0\right)$ и антиферромагнитного $\left(J_{2}<0\right)$ взаимодействия соответственно для первых и вторых ближайших соседей, $\theta_{i, j}, \theta_{i, k}-$ углы между взаимодействующими спинами $S_{i}-S_{j}$ и $S_{i}-S_{k}, H-$ величина магнитного поля (приводится в единицах $J_{1}$ ). В данном исследовании рассматривается случай, когда $\left|J_{1}\right|=\left|J_{2}\right|=1$. Величина внешнего магнитного поля менялась в интервале $0.0 \leq H \leq 3.0$ с шагом 1.0. Магнитное поле направлено вдоль одного из направлений спина.

Направления спинов заданы таким образом, что выполняется равенство

$$
\begin{aligned}
\theta_{i, j} & =\left\{\begin{array}{ll}
0 & \text { if } S_{i}-S_{j} \\
109.47^{\circ}, & \text { if } S_{i} \neq S_{j}
\end{array} \Rightarrow \cos \theta_{i j}\right. \\
& = \begin{cases}1 & \text { if } S_{i}=S_{j} \\
-1 / 3, & \text { if } S_{i} \neq S_{j} .\end{cases}
\end{aligned}
$$

Такие системы в настоящее время успешно изучаются на основе метода МК микроскопических гамильтонианов [15-20]. В последние годы разработано много новых алгоритмов метода МК. Одним из наиболее эффективных для исследования подобных систем являются репличный обменный алгоритм [21].

Репличный обменный алгоритм был использован нами в следующем виде:

1. Одновременно моделируются $N$ реплик $X_{1}, X_{2}, \ldots X_{N}$ с температурами $T_{1}, T_{2}, \ldots T_{N}$.

2. После выполнения одного МК-шага/спин для всех реплик производится обмен данными между парой соседних реплик $X_{i}$ и $X_{i+1}$ в соответствии со схемой Метрополиса с вероятностью

$$
w\left(X_{i} \rightarrow X_{i+1}\right)= \begin{cases}1, & \text { for } \Delta \leq 0 \\ \exp (-\Delta), & \text { for } \Delta>0\end{cases}
$$

где $\Delta=\left(U_{i}-U_{i+1}\right) \cdot\left(1 / T_{i}-1 / T_{i+1}\right), U_{i}$ и $U_{i+1}-$ внутренние энергии реплик.

Для анализа природы и характера ФП использовались метод кумулянтов Биндера четвертого порядка и гистограммный метод анализа данных [22-24]. Для вывода системы в состояние термодинамического равновесия отсекался участок длиной $\tau_{0}=4 \cdot 10^{5}$ шагов
МК на спин, что в несколько раз больше длины неравновесного участка. Усреднение термодинамических параметров проводилось вдоль марковской цепи длиной до $\tau=500 \tau_{0}$ шагов МК на спин. Расчеты проводились для систем с периодическими граничными условиями и линейными размерами $2 \times L \times L \times L=N, L=12 \div 60$, где $L-$ линейный размер решетки, $N-$ количество спинов в системе.

\section{3. Результаты моделирования}

Для наблюдения за температурным ходом поведения теплоемкости $C$ нами использовалось выражение [25]:

$$
C=\left(N K^{2}\right)\left(\left\langle U^{2}\right\rangle-\langle U\rangle^{2}\right)
$$

где $K=\left|J_{1}\right| / k_{B} T, U-$ внутренняя энергия.

На рис. 1 представлены температурные зависимости теплоемкости $C$ для различных значений магнитного поля при $L=24$. Из рисунка видно, что в интервале $0.0 \leq H \leq 3.0$ вблизи критической области наблюдаются хорошо выраженные максимумы. При включении слабого магнитного поля $(H=1.0)$ максимум теплоемкости смещается в сторону высоких температур. Дальнейший рост поля приводит к сдвигу максимума теплоемкости в сторону низких температур. Такое поведение теплоемкости объясняется тем, что увеличение величины магнитного поля приводит к быстрому упорядочению системы, уменьшению флуктуаций и соответственно уменьшается температура ФП. Для значений поля $H=2.0$ максимум теплоемкости становится более плавными. Можно предположить, что такое поведение теплоемкости связано с изменением магнитного упорядочения.

Для анализа характера ФП и определения критической температуры ТС нами использовался метод куму-



Рис. 1. Температурные зависимости теплоемкости $C / k_{B}$. 
лянтов Биндера четвертого порядка [22]:

$$
\begin{gathered}
V_{L}=1-\frac{\left\langle U^{4}\right\rangle_{L}}{3\left\langle U^{2}\right\rangle_{L}^{2}} \\
U_{L}=1-\frac{\left\langle m^{4}\right\rangle_{L}}{3\left\langle m^{2}\right\rangle_{L}^{2}}
\end{gathered}
$$

где $V_{L}-$ энергетический кумулянт, $U_{L}-$ магнитный кумулянт.

Параметр порядка системы $m$ вычислялся по формуле:

$$
m=\frac{1}{N}\left(\frac{4 N_{\max }-N_{1}-N_{2}-N_{3}-N_{4}}{3}\right),
$$

где $N_{1}, N_{2}, N_{3}, N_{4}$ - число спинов, соответствующих одному из 4 направлений спина соответственно.

Выражения (4) и (5) позволяют определить критическую температуру $T_{C}$ с большой точностью для ФП первого и второго рода соответственно. Применение кумулянтов Биндера позволяет также хорошо тестировать тип ФП в системе. Известно, что ФП первого рода характеризуются тем, что величина $V_{L}$ стремится к некоторому нетривиальному значению $V^{*}$ согласно выражению

$$
V_{L}=V^{*}+b L^{-d}
$$

при $L \rightarrow \infty$ и $T=T_{C}(L)$, где величина $V^{*}$ отлична от $2 / 3$, а минимальная величина $U_{L} \min \left(T=T_{\min }\right)$ расходится $\left(U_{L} \min (T=T \min ) \rightarrow \infty\right)$ при $L \rightarrow \infty$.

В случае ФП второго рода кривые температурной зависимости кумулянтов Биндера $U_{L}$ имеют четко выраженную точку пересечения [22].

На рис. 2 представлена характерная зависимость $U_{L}$ от температуры для поля $H=3.0$ при разных значениях $L$. Из графика видно, что в критической области температурные зависимости $U_{L}$ не пересекаются в одной точке. Это свидетельствует в пользу наличия в данной модели ФП первого рода. Аналогичная картина наблюдается для всех значений поля в диапазоне $0.0 \leq H \leq 3.0$.

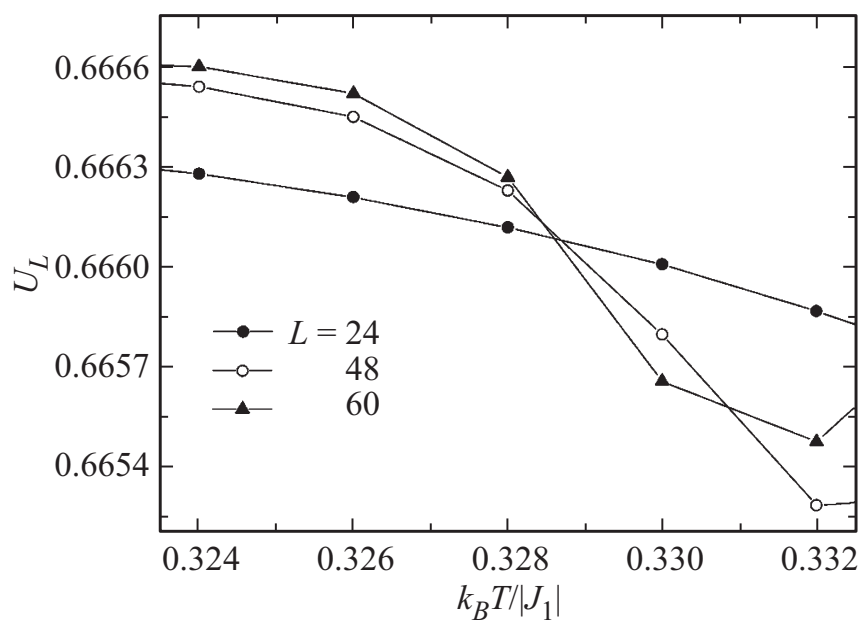

Рис. 2. Температурные зависимости магнитного кумулянта Биндера $U_{L}$.

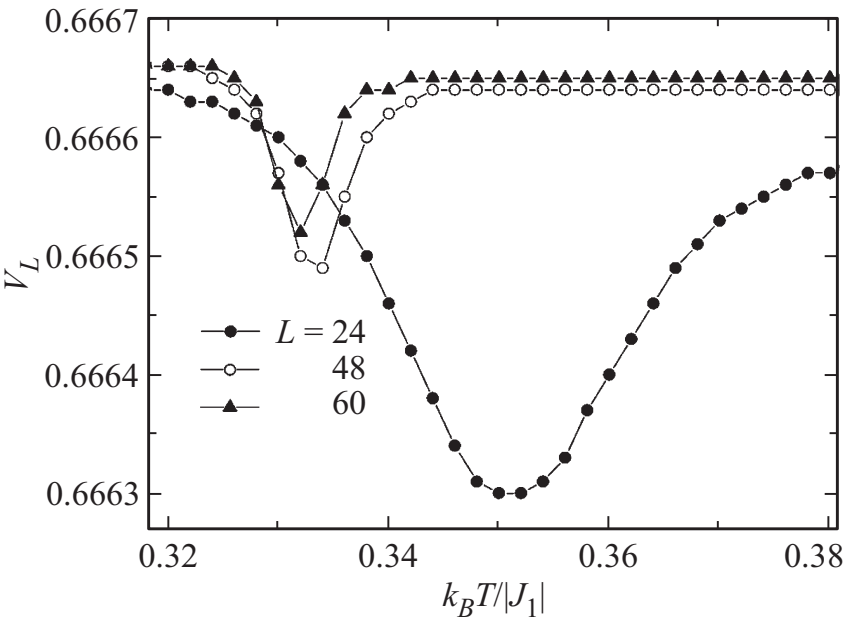

Рис. 3. Температурные зависимости энергетического кумулянта Биндера $V_{L}$.
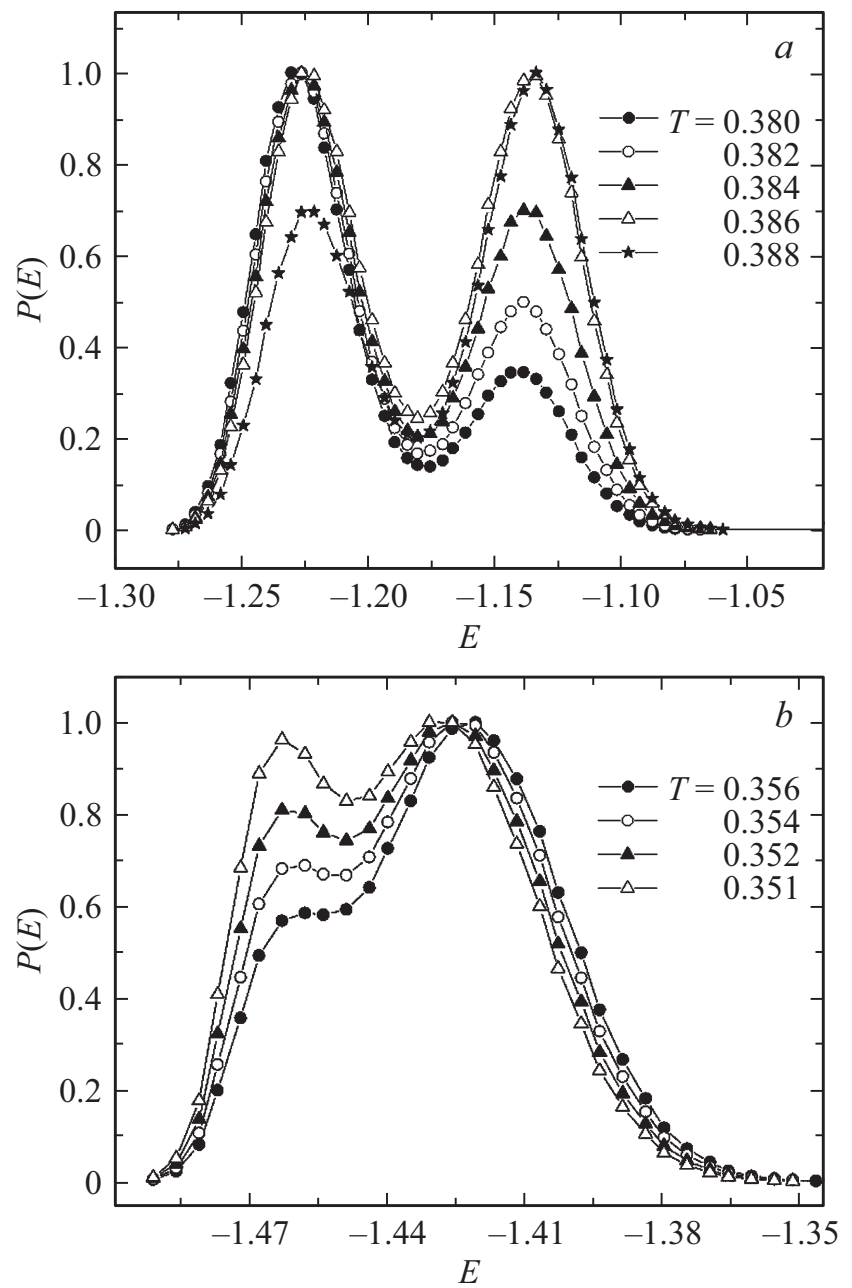

Рис. 4. Гистограммы распределения энергии для $L=60$ при различных температурах.

Температурная зависимость энергетического кумулянта $V_{L}$ для поля $H=3.0$ при разных значениях $L$ представлена на рис. 3. Как видно из графика величина $V_{L}$ 
стремится к $2 / 3$, а величина $V^{*}=2 / 3$, что характерно для ФП второго рода. Эта величина рассчитана используя выражение (7).

Для более подробного анализа рода ФП нами использовался гистограммный анализ данных метода МК $[23,24]$. Этот метод позволяет надежно определить род ФП. Методика определения рода ФП этим методом подробно описана в работе [17].

Полученные на основе гистограммного анализа данных результаты показывают, что в данной модели наблюдается ФП первого рода. Это продемонстрировано на рис. 4. На этом рисунке представлены гистограммы распределения энергии для системы с линейными размерами $L=60$ для $H=1.0$ и 2.0. Графики построены при различных температурах близких критической температуре. Из рисунка видно, что в зависимости вероятности $P$ от энергии $E$ для всех температур наблюдаются два максимума, которые свидетельствует о ФП первого рода. Наличие двойного пика на гистограммах распределения энергии является достаточным условием для ФП первого рода. Отметим, что двойные пики на гистограммах распределения для исследуемой модели наблюдаются для значений $H$ в интервале $0.0 \leq H \leq 3.0$. Это позволяет нам утверждать о том, что в рассмотренном интервале значений $H$ наблюдаются ФП первого рода.

Результаты данной работы показывают, что слабое внешнее магнитное поле не приводит к смене ФП в исследуемой модели. Исследование ФП данной модели в широком интервале значений $H$ в литературе не встречается.

\section{4. Заключение}

Исследование влияния слабого внешнего магнитного поля на фазовые переходы и термодинамические модели Поттса с числом состояний спина $q=4$ на гексагональной решетке с взаимодействиями вторых ближайших соседей выполнено с использованием репличного обменного алгоритма метода Монте-Карло. На основе гистограммного метода и метода кумулянтов Биндера проведен анализ характера фазовых переходов. Показано, что в интервале значений $0.0 \leq H \leq 3.0$ наблюдается фазовый переход первого рода.

\section{Конфликт интересов}

Авторы заявляют, что у них нет конфликта интересов.

\section{Список литературы}

[1] H.T. Diep. Frustrated Spin Systems. World Scientific Publishing Co. Pte. Ltd., Singapore (2004). P. 624.

[2] R.J. Baxter. Exactly Solved Models in Statistical Mechanics. Academic, N. Y. (1982). Mir, Moscow( 1985).

[3] F.Y. Wu. Exactly Solved Models: A Journey in Statistical Mechanics. World Scientific, New Jersey( 2008).
[4] F.Y. Wu. Rev. Mod. Phys. 54, 235 (1982).

[5] W. Zhang, Y. Deng. Phys. Rev. E 78, 031103 (2008).

[6] А.К. Муртазаев, М.К. Рамазанов, Ф.А. Кассан-Оглы, М.К. Бадиев. ЖЭТФ 144, 1239 (2013).

[7] A.K. Murtazaev, M.K. Ramazanov, M.K. Badiev. Phys. B: Condens. Matter 476, 1 (2015).

[8] F.A. Kassan-Ogly, A.K. Murtazaev, A.K. Zhuravlev, M.K. Ramazanov, A.I. Proshkin. J. Magn. Magn. Mater. 384, 247 (2015).

[9] M. Nauenberg, D.J. Scalapino. Phys. Rev. Lett. 44, 837 (1980).

[10] J.L. Cardy, M. Nauenberg, D.J. Scalapino. Phys. Rev. B 22, 2560 (1980).

[11] M.K. Ramazanov, A.K. Murtazaev, M.A. Magomedov. Phys. A 521, 543 (2019).

[12] H. Feldmann, A.J. Guttmann, I. Jensen, R. Shrock, S.-H. Tsai. J. Phys. A 31, 2287 (1998).

[13] F.A. Kassan-Ogly, A.I. Proshkin. Phys. Solid State 60, 1090 (2018).

[14] H. Feldmann, A.J. Guttmann, I. Jensen, R. Shrock, S.-H. Tsai. J. Phys. A 31, 2287 (1998).

[15] А.К. Муртазаев, М.К. Рамазанов, М.К. Мазагаева, М.А. Магомедов. ЖЭТФ 156, 502 (2019).

[16] М.К. Рамазанов, А.К. Муртазаев, М.А. Магомедов, М.К. Мазагаева. ФТТ 62, 442 (2020).

[17] М.К. Рамазанов, А.К. Муртазаев. Письма в ЖЭТФ 109, 610 (2019).

[18] А.К. Муртазаев, М.К. Рамазанов, М.К. Бадиев. ФТТ 61, 1898 (2019).

[19] А.К. Муртазаев, Д.Р. Курбанова, М.К. Рамазанов. ФТТ 61, 2195 (2019).

[20] R. Masrour, A. Jabar. Physica A 491, 926 (2018).

[21] A. Mitsutake, Y. Sugita, Y. Okamoto. Biopolymers (Peptide Science) 60, 96 (2001).

[22] K. Binder, D. Heermann. Monte Carlo Simulation in Statistical Physics: An Introduction. Springer, Berlin, Heidelberg( 2010).

[23] F. Wang, D.P. Landau. Phys. Rev. E 64, 0561011-1 (2001).

[24] F. Wang, D.P. Landau. Phys. Rev. Lett. 86, 2050 (2001).

[25] P. Peczak, A.M. Ferrenberg, D.P. Landau. Phys. Rev. B 43, 6087 (1991).

Редактор Т.Н. Василевская 